\title{
THE FUNDAMENTAL THEOREM OF ALGEBRA ON RATIONAL $H$-SPACES
}

\author{
LAURA WEISS ${ }^{1}$
}

\begin{abstract}
A form of the Fundamental Theorem of Algebra is proven for r. $H$ structures.
\end{abstract}

0 . Introduction and summary of results. It is a curiosity that while the Fundamental Theorem of Algebra is algebraic in content and statement, its proof is topological. We show here that the structure of a rational $H$-space, or $\Gamma$ structure is sufficient to prove a form of the theorem.

In $\S 1$ we construct a rational $H$-space structure on the Stiefel manifold $V_{5,2}$ which we believe has not been explicitly exhibited before. In $\S 2$ we prove that any homotopy associative rational $H$-space is actually an $H$-space.

1. Rational $H$-spaces or $\Gamma$ structures. A rational $H$-space is a triple $(X, m, e)$ consisting of a connected space $X$, a product map $m: X \times X \rightarrow X$ which preserves the basepoint $e$, and which satisfies the following condition. Let $\varphi_{i}: X \rightarrow X \times X$ be inclusion into the $i$ th factor, $i=1,2$. Then $m_{l}=m \varphi_{1}$ and $m_{r}=m \varphi_{2}$ must induce automorphisms of $H^{*}(X ; \mathbf{Q})$.

If $m_{l} \simeq m_{r} \simeq$ id, then $e$ is a homotopy identity and $(X, m, e)$ is actually an $H$-space.

For notational simplicity we will follow Hopf [4] and refer to rational $\boldsymbol{H}$ spaces as $\Gamma$ structures.

Examples. (1) (Hopf [4]). For an odd dimensional sphere define $m: S^{n} \times S^{n}$ $\rightarrow S^{n}$ by $m(p, q)=q$ reflected through the orthogonal complement of $p$. Then $m_{r}^{*}(z)=-z$ and $m_{l}^{*}(z)=2 z$ where $z$ generates $H^{*}\left(S^{n}\right)$.

(2) Consider the Stiefel manifold $V_{n, 2}$ of 2 frames in $\mathbf{R}^{n}$ for odd $n$. $V_{n, 2}$ can be fibered as an $n-2$ sphere bundle over $S^{n-1}$ [5]. Since $\Pi_{2 n-3}\left(S^{n-1}\right)$ has a cyclic infinite subgroup [3, p. 74], and $\Pi_{k}\left(S^{n-2}\right)$ is finite whenever $k \neq n-2$ [7, p. 515], it is clear from the long exact sequence of the fibration that $\mathrm{II}_{2 n-3}\left(V_{n, 2}\right)$ has a cyclic infinite subgroup, with generator, say $g$.

Let $\mu$ be a generator of $H^{2 n-3}\left(S^{2 n-3}, \mathbf{Z}\right)$. Then $g_{*}(\mu)=h([g])$ where $h: \Pi_{2 n-3}\left(V_{n, 2}\right) \rightarrow H_{2 n-3}\left(V_{n, 2} ; \mathbf{Z}\right)$ is the Hurewicz homomorphism. Since $H^{*}\left(V_{n, 2} ; \mathbf{Q}\right)=\Lambda\left(z_{2 n-3}, 1\right)$, an exterior algebra on odd dimensional generators [6], we can apply a result of Arkowitz and Curjel [1] to conclude that $g^{*}(\mu) \neq 0$.

The cell structure of $V_{n, 2}$ is known to be $S^{n-2} \cup e^{n-1} \cup e^{2 n-3}$. Let $\rho: V_{n, 2}$

Received by the editors August 24, 1974 and, in revised form, November 4, 1974.

AMS (MOS) subject classifications (1970). Primary 55D45.

Key words and phrases. Rational $H$-space, $\Gamma-H$ structure, Stiefel manifold $V_{n, 2}$, Fundamental Theorem of Algebra.

${ }^{1}$ Supported by graduate assistantship at the University of Rochester. 
$\rightarrow S^{2 n-3}$ be the collapsing map. Then the composition

$$
V_{n, 2} \times V_{n, 2} \stackrel{\rho \times \rho}{\longrightarrow} S^{2 n-3} \times S^{2 n-3} \stackrel{m}{\longrightarrow} S^{2 n-3} \stackrel{g}{\longrightarrow} V_{n, 2}
$$

is a $\Gamma$ structure on $V_{n, 2}$ where $m$ is Hopf's $\Gamma$ structure.

It is well known that if a CW complex $X$ admits a $\Gamma$ structure, then $H^{*}(X, \mathbf{Q})=\Lambda_{\nu \in N}\left\{z_{\nu}\right\} \otimes P_{\mu \in M}\left\{x_{\mu}\right\}$ where $\Lambda\left\{z_{\nu}\right\}$ is an exterior algebra on odd dimensional generators and $P\left\{x_{\mu}\right\}$ is a polynomial algebra on even dimensional generators. Furthermore, for $y \in H^{*}(X, \mathbf{Q})$,

$$
m^{*}(y)=m_{l}^{*}(y) \otimes 1+\sum y^{\prime} \otimes y^{\prime \prime}+1 \otimes m_{r}^{*}(y)
$$

with $\operatorname{deg} y^{\prime}>0$.

2. Words on $\Gamma$ structures. The product $m$ induces a binary operation, called convolution, on the set of maps from $X$ to itself. $f \circ g=m \circ f x g \circ \Delta$ where $\Delta(x)=(x, x)$, the usual diagonal. A word on $X$ is the convolution of a finite number of identity and constant maps. The identity map on $X$ will be denoted by $\iota$, the constant map at $p$ by $\omega_{p}$ or simply $\omega$. Other identity maps will be denoted by id.

THEOREM 1.1. If $(X, m, e)$ is a homotopy associative $\Gamma$ structure, then $X$ admits an $H$ structure.

Proof. It suffices to show that $m_{r}^{*}=m_{l}^{*}=\mathrm{id}$. Then, by Whitehead's theorem, $m_{r}$ and $m_{l}$ are homotopy equivalences and $m \circ\left(m_{l}^{-1} \times m_{r}^{-1}\right)$ is an $H$ structure.

If $m$ is homotopy associative, then, in particular, $(\omega \cdot \omega)^{*} \cdot \iota=\omega^{*} \cdot(\omega \cdot \iota)^{*}$. Direct computation shows that the left-hand side is $m_{r}^{*}$ and the right side is $m_{r}^{*} \circ m_{r}^{*}$. A similar computation holds for $m_{l}^{*}$.

We adopt the technical convention that all words will be spelled correctly. That is, convolution is always from the left, i.e.,

$$
g_{1} \cdot g_{2} \cdot \ldots \cdot g_{r}=g_{1} \cdot\left(g_{2} \cdot\left(\cdots\left(g_{r-1} \cdot g_{r}\right)\right) \cdots\right)
$$

and no two consecutive maps are constants. (This is always possible since $\left.\omega_{p} \cdot \omega_{q}=\omega_{m(p, q)}\right)$

Let $\left\{y_{j}\right\}$ denote the canonical basis for $H^{*}(X ; \mathbf{Q})$ as a vector space over $\mathbf{Q}$ corresponding to $\left\{z_{\nu}\right\}_{\nu \in N}$ and $\left\{x_{\mu}\right\}_{\mu \in M}$. A typical element is of the form $y_{j} z_{\nu_{1}} z_{\nu_{2}} \cdots z_{v_{k}} x_{\mu_{1}} \cdots x_{\mu_{r}}$. For each basis element $y_{j}$ we define an integer

$$
\lambda\left(y_{j}\right)=\max \left\{\text { degree } z_{\nu_{i}} \text {, degree } x_{\mu_{j}}, i=1, \ldots, k ; j=1, \ldots, r\right\} .
$$

Let $G(X)$ be the vector space over $\mathbf{Q}$ spanned by $\left\{z_{\nu}\right\}_{\nu \in N} \cup\left\{x_{\mu}\right\}_{\mu \in M}$. Define $\alpha\left(z_{\nu}\right)$ and $\beta\left(z_{\nu}\right)$ to be the summand of $m_{l}^{*}\left(z_{\nu}\right)$ and $m_{r}^{*}\left(z_{\nu}\right)$ which lives in $G(X)$, i.e., if $m_{l}^{*}\left(z_{\nu}\right)=\sum q_{i} z_{\nu_{i}}+\sum a_{j} y_{j}, a_{i} a_{j} \in \mathbf{Q}$ and $\lambda\left(y_{j}\right)<\operatorname{degree} z_{\nu}$, then $\alpha\left(z_{\nu}\right)=\sum a_{i} z_{\nu_{i}}$.

Associated to every word $f$ on $X$ we define an automorphism of $G(X)$ called the exponent of $f, \epsilon(f)$.

$$
\begin{aligned}
\epsilon(f) & =\text { id } & & \text { if } f=\iota, \\
& =0 & & \text { if } f=\omega, \\
& =\alpha+\epsilon(g) \circ \beta & & \text { if } f=\iota \cdot g, \\
& =\epsilon(g) \circ \beta & & \text { if } f=\omega \cdot g .
\end{aligned}
$$


If $(X, m, e)$ is an $H$-space, then $\alpha=\beta=$ id and $\epsilon(f)$ coincides with the definition of exponent given by R. F. Brown [2].

Lemma 2.1. If $f: X \rightarrow X$ is a word, then $f^{*}\left(z_{\nu}\right)=\epsilon(f) z_{\nu}+\sum a_{j} y_{j}$ where $\lambda\left(y_{j}\right)<$ degree $z_{\nu}$.

Proof. The result is clear if $f=\imath$ or $\omega$. We proceed by induction on the number of maps convoluted to form $f$. If $f=\imath \cdot g$, then $f^{*}\left(z_{v}\right)=\alpha\left(z_{v}\right)$ $+\sum a_{j} y_{j}+g^{*} \beta\left(z_{\nu}\right), \beta\left(z_{\nu}\right)=\sum b_{i} z_{v_{i}}, b_{i \in \mathbf{Q}}$, degree $z_{v_{i}}=$ degree $z_{v}$. Hence

$$
\begin{aligned}
g^{*} \beta\left(z_{\nu}\right) & =\sum b_{i} g^{*}\left(z_{\nu_{i}}\right)=\sum b_{i}\left(\epsilon(g) z_{\nu_{i}}+\sum a_{j} y_{j}\right) \\
& =\epsilon(g) \circ \beta\left(z_{\nu}\right)+\sum a_{j} y_{j}
\end{aligned}
$$

with $\lambda\left(y_{j}\right)<$ degree $z_{v}$. The case $f=\omega \cdot g$ is a similar computation.

3. $\Gamma$ - $H$ structures. Following [2] we define $C^{0}(X)$, the open cone on $X$, as $X \times[0, \infty)$ with $X \times\{0\}$ collapsed to a point. Square brackets will be used to denote equivalence classes and $[x, 0]$ will be written as 0 . Any $\Gamma$ structure $(X, m, e)$ can be extended to a $\Gamma$ structure $\left(C^{0}(X), c(m),[e, 1]\right)$ by defining $c(m)([x, t],[y, s])=[m(x, y), t s]$.

A $\Gamma-H$ structure $(X, m, e, \eta)$ is a $\Gamma$ structure $(X, m, e)$ together with an $H$ structure on $C^{0}(X)$ for which 0 is the basepoint, i.e., $\eta: C^{0}(X) \times C^{0}(X)$ $\rightarrow C^{0}(X)$ and $\eta([x, t], 0)=\eta(0,[x, t])=[x, t]$. We will denote the binary operation $\eta$ induces on words of the $\Gamma$ structure $\left(C^{0}(X), c(m),[e, 1]\right)$ by $q$. A polynomial on $C^{0}(X)$ is a map from $C^{0}(X)$ to itself of the form $g_{1} \uparrow g_{2} \uparrow \ldots$ $q g_{k}$, where $g_{i}$ is a word on $\left(C^{0}(X), c(m),[e, 1]\right) .[x, t]$ is a root of a polynomial if $f[x, t]=0$.

EXAMPLE 3.1. Let $X=S^{1}$ considered as complex numbers of unit norm, $m$ is standard complex multiplication, $\eta$ is usual addition. $\omega_{a_{n}} \cdot \iota^{n} \uparrow \omega_{a_{n-1}} \cdot \iota^{n-1}$ $q \cdots q \omega_{a_{0}}$ is the familiar polynomial $a_{n} x^{n}+a_{n-1} x^{n-1}+\cdots+a_{0}$.

Given a polynomial $f=g_{1} \uparrow g_{2} \uparrow \cdots \uparrow g_{k}$, define a polynomial

$$
f_{i s}=\psi\left(s, g_{1}\right) q \cdots q \psi\left(s, g_{i-1}\right) q g_{i} \uparrow \psi\left(s, g_{i+1}\right) q \cdots \uparrow \psi\left(s, g_{k}\right),
$$

i.e. apply $\psi(s$,$) to each word except g_{i}$ where

$$
\psi(s, g)[x, t]=\theta(s, g[x, t]) \text { and } \theta(s,[x, t])=[x, s t] .
$$

The polynomial $f$ is admissible if $f_{i, s}$ is proper for some $i$ uniformly in $s$, i.e. $f_{i, s}$ extends to the suspension $S X$ viewed as the one point compactification of $C^{0}(X)$. Not all nonconstant words are admissible.

EXample 3.2. Consider the quaternions as $C^{0}\left(S^{3}\right)$. Then the polynomial $\omega_{[i, 1]} \uparrow \imath \cdot \omega_{[i, 1]} \uparrow \omega_{[i, 1]} \cdot \imath$ is not admissible and does not have a root.

LEMMA 3.3. If $f=g_{1} q \cdots q g_{k}$ is an admissible polynomial with $f_{i, s}$ proper, then $\tilde{f} \simeq \tilde{g}_{i}$, where tilde denotes the extension to $S X$.

Proof. Define $H: S X \times I \rightarrow S X$ by $H([x, t], s)=\tilde{f}_{i s}[x, t]$.

A word $g$ on $(X, m, e)$ is compatible with $m$ if $\epsilon(g) \neq 0$. A polynomial $f=g_{1} \uparrow \cdots q g_{k}$ is compatible if $f$ is admissible and $g_{i}$ is compatible with $c(m)$. Define a word $\bar{g}_{i}$ on $(X, m, e)$ by $\bar{g}_{i}(x)=g_{i}[x, 1]$. Then by $3.3, \tilde{f} \simeq \tilde{g}_{i} \simeq \sum \bar{g}_{i}$. 
EXAmPLe 3.4. Let $m: S^{1} \times S^{1} \rightarrow S^{1}$ be given by $m\left(e^{i \theta}, e^{i \varphi}\right)=e^{i(\theta-\varphi)}$. The polynomial $\iota^{2} \& \omega_{\left[e^{i \pi / 2}, 1\right]}$ is admissible but not compatible. In this case $g_{i}=\iota^{2}$

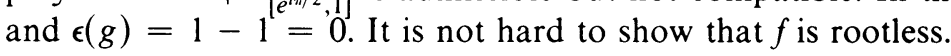

\section{Fundamental Theorem of Algebra.}

TheOREM. $(X, m, e, \eta)$ is a $\Gamma$ - $H$ structure with $H^{*}(X ; \mathbf{Q})=\Lambda\left\{z_{v}\right\} \otimes P\left\{x_{\mu}\right\}$. Every polynomial on $C^{0}(X)$ which is compatible with $m$ has a root.

Proof. Suppose $f=g_{1} \subsetneq g_{2} q \cdots q g_{k}$. By compatibility $\tilde{f} \simeq \bar{g}_{i}$ and $\epsilon\left(\bar{g}_{i}\right)$ $\neq 0 . \quad \bar{g}_{i}^{*}\left(z_{v}\right)=\epsilon\left(\bar{g}_{i}\right) z_{\nu}+\sum a_{j} y_{j} \neq 0$ by Lemma 2.1. Then $\tilde{f}^{*} \sigma\left(z_{\nu}\right)$ $=S \bar{g}_{i}^{*}\left(\sigma z_{\nu}\right)=\sigma \bar{g}_{i}^{*}\left(z_{\nu}\right) \neq 0$ where $\sigma: H^{*}(X) \rightarrow H^{*}(S X)$ is the suspension homomorphism.

On the other hand if $\tilde{f}$ is not onto then its image will be contained in a contractible space and $\tilde{f}^{*}=0$.

Note this generalizes Theorem 2 of [2].

\section{REFERENCES}

1. M. Arkowitz and C. R. Curjel, Zum Begriff des H Raumes mod Fु, Arch. Math. 16 (1965), 186-190. MR 31 \#4031.

2. R. F. Brown, Words and polynomials in H-spaces, Amer. J. Math. 96 (1974), 229-236.

3. P. J. Hilton, An introduction to homotopy theory, Cambridge Tracts in Math. and Math. Phys., no. 43, Cambridge Univ. Press, New York, 1953. MR 15, 52.

4. Heinz Hopf, Über die Topologie der Gruppen-Mannigfaltigkeiten und ihre Verallgemeinerungen, Ann. of Math. (2) 42 (1941), 22-52. MR 3,61.

5. I. M. James and J. H. C. Whitehead, The homotopy theory of sphere bundles over spheres. I, Proc. London Math. Soc. (3) 4 (1954), 196-218. MR 15, 892.

6. Clair E. Miller, The topology of rotation groups, Ann. of Math. (2) 57 (1953), 90-114. MR 14, 673.

7. Edwin Spanier, Algebraic topology, McGraw-Hill, New York, 1966. MR 35 \# 1007.

Department of Mathematics, State University of New York, College at Potsdam, POTSDAM, NEW YORK 13676 